\title{
Adam P. Showman
}

\author{
Exoplanetary and Solar System science lost a major figure with the sudden passing of Professor Adam Showman \\ on 16 March 2020 at the age of 51. He was recognized as the world's leading authority in the field of atmospheric \\ dynamics of exoplanets.
}

A dam grew up in Cupertino, CA, and attended the public schools there while feeding his passion for science by exploring nature in his neighbourhood. A family trip to China while Adam was a teenager ignited a lifelong interest in the Chinese language and culture. After Adam received his BS in physics from Stanford University, he spent six months at Wuhan in Hubei province, China, teaching English, and another several months touring parts of Asia. For his $1999 \mathrm{PhD}$ at Caltech's Division of Geological and Planetary Sciences, Adam led efforts that had a direct bearing on the most exciting planetary science of the day: NASA's Galileo Mission to Jupiter. His graduate work was on two diverse fronts. The first was the tidal and structural evolution of Jupiter's large moon Ganymede, and how a past orbital resonance could have led to tidal heating that resurfaced the moon. The second was a detailed exploration of the physical effects that could give rise to the unexpected results from the Galileo Entry Probe, which dove into Jupiter's atmosphere. Adam's initial postdoctoral work at the University of Louisville expanded on his work on the Jovian atmosphere, detailing the still-leading theory for the structure of Jupiter's 5- $\mu \mathrm{m}$ 'hot spots'.

The discovery of the first transiting 'hot Jupiter' exoplanet in 1999, and the prospect of understanding the atmospheres of this class of planets, led Adam on a new research path while a postdoc at the NASA Ames Research Center. This new path would become central to his science legacy. In two seminal papers with Tristan Guillot in 2002, Adam, then a new assistant professor in Arizona, outlined the major aspects of the physics of the three-dimensional (3D) atmospheric circulation of hot Jupiters and how atmospheric dynamics could have a major impact on the thermal and structural evolution of these planets. The papers were extremely prescient regarding a range of atmospheric physics, particularly in predicting the nature of the circulation for these highly irradiated worlds, which has been gradually uncovered via observational work over the past two decades.

As a professor at the Lunar and Planetary Laboratory (LPL) at the University of

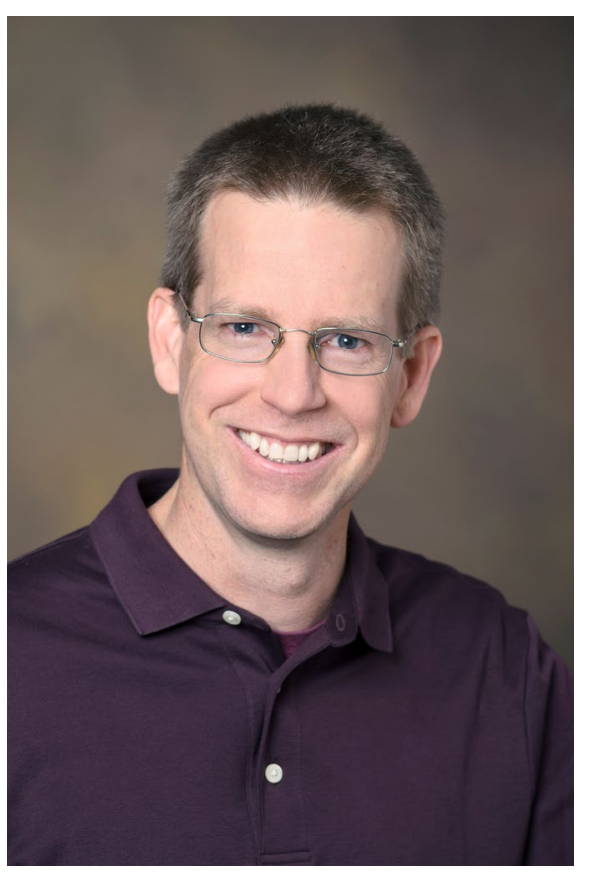

Adam P. Showman (1968-2020). Credit: University of Arizona

Arizona, Adam taught graduate-level classes in both planetary atmospheres and planetary tectonics, while also teaching specialized atmospheric dynamics classes in atmospheric sciences. He was known for his textbook-quality lecture notes, and the field has surely missed out on Adam someday turning those notes into insightful books. Adam wanted total understanding of the topics that he taught, which often led to very-late-night mastery of material before he was ready to present to his students. Adam also led the LPL graduate-student field trips for many years, planning and running field trips across the American Southwest.

As an advisor and mentor to $13 \mathrm{PhD}$ students (including three currently) and five postdoctoral scholars, who now work as university faculty (at NASA and the USGS) and in industry, Adam's research grew in breadth and depth. He continued his long-term interests in Solar System science and delivered groundbreaking results. In satellite geophysics, Adam and his students focused on the tectonics of Ganymede,
Titan and Enceladus. He greatly expanded this work to understand a wide range of aspects of the physics of solid-state convection in icy moons. He liked to make connections between higher-density geophysical fluid dynamics and atmospheric dynamics, pointing out that the equations were the same, just with different terms becoming dominant depending on the situation. In atmospheres, Adam's group showed that deep zonal flows can result from shallow forcing - heat sources and sinks near the surface. As was typical of Adam, this was demonstrated with models of increasing complexity, ranging from thin-shell models to fully 3D models of a fluid planet. The deep winds of Jupiter are a hot topic yet again today with NASA's Juno Mission, and Adam was involved in developing some of the pioneering methods used for the Juno detections. Adam and his group also developed the first Global Circulation Models (GCMs) that could consistently reproduce the zonal jets on all four giant planets.

As the exoplanet field matured, so did Adam's point of view. Across several years, he led the development of a sophisticated GCM for hot Jupiter atmospheres, published in 2009, that enabled direct comparison of these 3D models with emerging observations from the Spitzer and Hubble Space Telescopes. This model was a dramatic leap in the state-of-the-art that was not equalled for nearly a decade. Adam's interest in the comparison with observations led to many collaborations on telescope proposals with the world's leading exoplanet observers.

While Adam was the founder of a wide swathe of the field of exoplanetary atmospheric dynamics, what is perhaps even more impressive is that he maintained his role as the essential character in this field up until his untimely death. He and his group demonstrated the role of atmospheric dynamics in altering the emitted and reflected spectra compared to simple models, driving non-equilibrium chemical abundances, and driving particle transport vertically and horizontally. Over the past several years Adam and his group were pioneering more groundbreaking work in different physical regimes, including understanding the atmospheric dynamics of 
brown dwarfs and directly imaging planets on wide-separation orbits.

A thread throughout Adam's life was his continued interest and love of China and its culture. Initially through group classes, then one-on-one language practice, and later through marriage, Adam became fluent in speaking Mandarin to the extent that he could give scientific seminars, and strong in writing the language as well. In 2011, Adam was invited to deliver a series of talks at the Kavli Institute for Astronomy and Astrophysics at Peking University, and he stayed at the Institute all that summer. Adam visited the Department of Atmospheric and Oceanic Sciences of the School of Physics at Peking University most summers in the past decade, working with faculty and students, in addition to a 2016-2017 year-long sabbatical.
Adam always had a rare curiosity to learn new things. This characteristic led him on nature walks as a child and continued to drive his personality as an adult. In addition to his Chinese language work he practised oil painting with some amazing successes. At Caltech he revived the ballroom dance club and was later an avid swimmer. A key aspect of his personality is that while he was serious in his science, Adam was always approachable and never took himself too seriously. As was well known to many early career scientists, Adam was a frequent participant of the LPL graduate-student-led Christmas skits, which are often a send-up of graduate-student life and pop culture. We can hope that the magnum opus of these skits, 'Showman Style, lives forever on YouTube, as it is a real reminder that one can be a giant in one's field and still save plenty of time to have fun.
The hallmark of Adam's scientific skill was his ability to take apart extremely complex problems in an effort to come to an understanding of the underlying physical mechanisms. Very few have done this as well. The excellence of Adam's scientific contributions was recently recognized with his selection as a Fellow of the American Geophysical Union in 2019. Adam's students, mentees, and collaborators all learned a great deal from him, and many fields of planetary sciences were enriched with his presence. Adam will be greatly missed.

Jonathan J. Fortney

University of California, Santa Cruz, CA, USA.

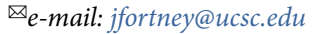

Published online: 27 April 2020

https://doi.org/10.1038/s41550-020-1107-2 